\title{
COMMUNICATIONS
}

\section{EFFECT OF AREA AND INTENSITY ON THE SIZE AND SHAPE OF THE ELECTRORETINOGRAM* EXCLUSION OF STRAY LIGHT EFFECTS}

\author{
BY \\ ALBERTO WIRTH and BIRGITTA ZETTERSTRÖM \\ Nobel Institute for Neurophysiology, Karolinska Institutet, Stockholm, Sweden
}

SiNCE Adrian and Matthews (1928) showed that the latent period of the optic nerve discharge can be influenced by the size of the area stimulated, the effect of area on the electroretinogram has been confirmed by a number of workers (Granit, 1933; Creed and Granit, 1933; Fry and Bartley, 1935; Boynton and Riggs, 1951)،

The size and shape of the electroretinogram can be influenced in the same way by increasing the stimulated area as by increasing the intensity of the stimulus. Fry and Bartley (1935) stated that the effect of area was due almost entirely to stray light; they believed the effect of the focal area to be small. They performed the following experiment on the intact rabbit eye:

Two adjacent bright areas were exposed alternately against a dark background. Since the amount of stray light was the same in each case and diffusely distributed over the retina, alteration could not affect it: each of the two retinal spots, however, should respond intermittently if the focal response were dominant. Actually, at fast rates of alternation, no response was obtained, and this finding was explained on the assumption that stray light, being constant and independent of the area stimulated, acted as a much more effective constant background than the alternating focal points, which were therefore believed to elicit only a negligible fraction of the $b$-wave.

Granit, Rubinstein, and Therman (1935) disagreed with this generalization. To test it they produced, on the retina of the excised and opened frog eye, two sharply focused spots, about $0.4 \mathrm{~mm}$. in diameter and $0.3 \mathrm{~mm}$. apart.

Threshold stimuli were used to elicit a response of about $50 \mu \mathrm{V}$. In this case the two stimuli $A$ and $B$ together elicited a response only 10-15 per cent. less than the expected value of $\mathbf{A}+\mathbf{B}$. Stray light could thus account for only this percentage of the combined response. Nevertheless when one flash was made to succeed the other, the effect of the second was inhibited. They concluded that adjacent neurones also were influenced by some kind of physiological spread of the electrical response, while admitting of course the existence of stray light unless special precautions were taken to avoid it.

More recently Boynton and Riggs (1951) and Asher (1951) have emphasized that the retinal response is primarily aroused by stray light rather than

*Received for publication November 6, 1953 
by the focal area. If the stimulus is made to fall on to the blind spot, there is no significant difference between the response obtained and that elicited by stimulation of a nearby area of equal size. Their view appears to be that any effect of stimulus area on the electroretinogram is doubtful.

Our problem was to study the electroretinogram by a device completely excluding stray light at high intensities with the stimulated area varying in size. We also wished to ascertain the minimal area necessary for the appearance of a standard electroretinogram. In view of the practical application of electroretinography to clinical problems, information on this point has become highly desirable.

\section{Method}

Dark-adapted cats were used. They: were anaesthetized with Nembutal $40 \mathrm{mg} . / \mathrm{kg}$. intraperitoneally, 4-6 ml. urethane 20 per cent. solution being added if needed. After removal of cornea and lens, the iris was cut radially to increase the size of the pupil and part of the vitreous body was gently removed. From a Philips tungsten band lamp run off high capacity storage batteries at a constant temperature of $2,800^{\circ} \mathrm{K}$, a parallel beam was focused by means of a mirror and a lens into the opened eye. Ilford neutral filters were used to vary stimulus intensity. Silver silver-chloride electrodes ending in a cotton wick soaked in saline were applied to the limbus and nose of the animal, and the leads were taken to a condenser-coupled amplifier of long time constant and a cathode-ray tube. The sweep was synchronized with a photocell. Single flashes 1/10 sec. in duration obtained with the aid of an Ibsor shutter were given at 30 -sec. intervals $(60 \mathrm{sec}$. at maximal intensity). To avoid stray light on the retina special Perspex cones 1-2-3-5 mm. in diameter at the top were used, and their external surface was blackened in order to prevent leakage of light. Selection of these particular values was based on preliminary experiments. Calibration revealed that the amount of light per unit area was the same, whether the whole pupil was filled or one of the different cones was used.

After not less than $40 \mathrm{~min}$. dark adaptation the experiment was begun by taking a control electroretinogram at maximum intensity $(2,000-3,000$ lux, the exact value determined in each case) with light filling the whole pupil. Then a cone was inserted and moved step by step by a Zeiss micromanipulator down to the retinal surface; records were then taken at different intensities. The experiment was discarded as soon as the retina lost sensitivity with reference to the control response with the cone absent. This often occurred through bleeding and retinal detachment.

To judge how far the cones had to be moved into the eye in order to reach the retinal surface, red marks were painted on them at different levels: preliminary adjustments were made in the light by observing the depth at which the cone was close to the retinal surface. It sometimes happened that the cone was pushed a little too deep; then the electroretinogram rapidly diminished, but it recovered its normal size when the cone was slightly raised.

In some experiments we used intermittent illumination obtained by means of a rotating sectored disk providing equal periods of light and darkness. The disk was turned by a Velodyne reversing motor generator (British Air Ministry type 74) controlled electronically (Enroth, 1952). Fusion frequency was measured with a resonance method, as described by Granit and Wirth (1953). A resonance meter (vibration analyser, made by the General Radio Corporation, U.S.A.) was set to respond to the required frequency, the flickering electroretinogram being recorded and indicated by the galvanometer needle of the resonance meter. As long as flicker was present resonance was obtained on this instrument. Disappearance of resonance signified fusion (see below). 


\section{Results}

Records from one experiment are presented in Fig. 1: only the initial phases of the electroretinogram were recorded since these were the ones used for quantitative analysis. The electroretinograms were taken at different intensities with the light filling the pupil and with retinal spots 5 and $3 \mathrm{~mm}$. in diameter. On the left a complete series shows normal electroretinograms over a wide range of intensities. Only at maximum intensity (2,050 lux) was a definite $a$-wave present. The $b$ wave then rose very steeply and exhibited an initial faster component. As the intensity was decreased, the amount of potential was less influenced to begin with than the rate of rise and the latent period. At low intensities the $b$-wave flattened out and its latency lengthened. On the right a number of records taken with restricted areas are presented for comparison. The most striking feature is that the size and shape of the electroretinogram were more abruptly affected by a decrease in intensity of

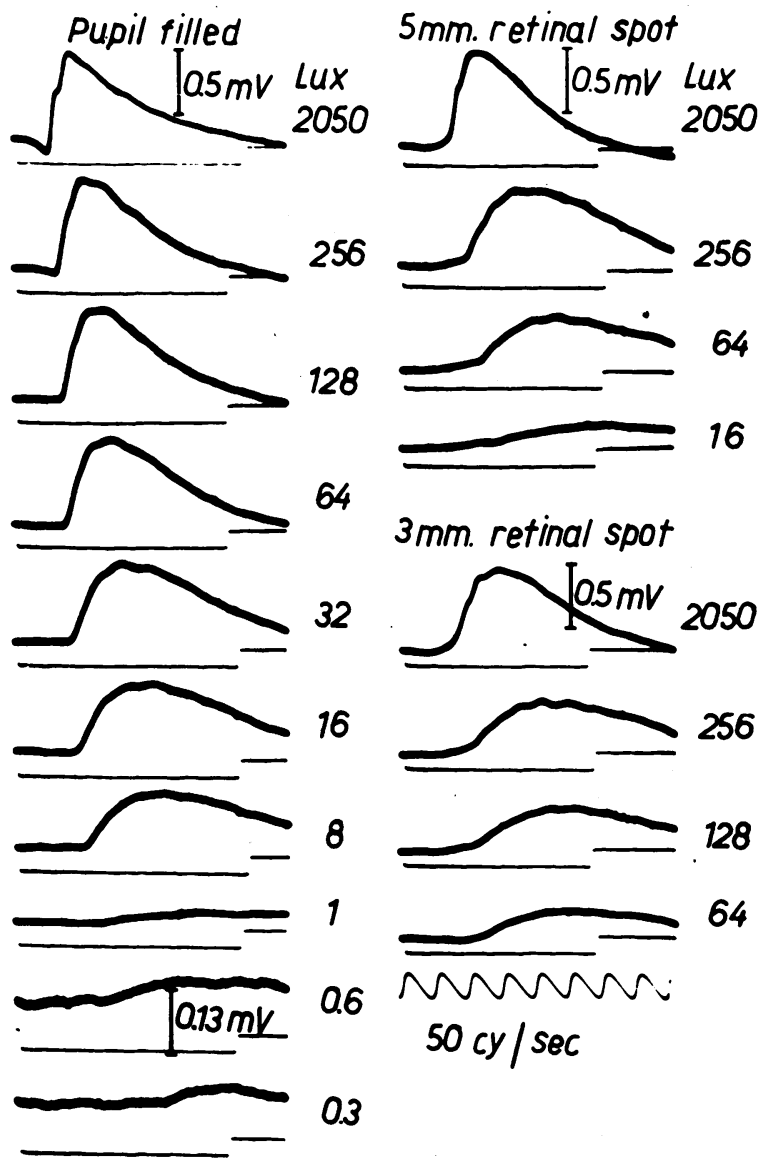

FIG. 1.-Electroretinograms recorded with cathode ray oscillograph at different intensities, with light filling the pupil, and with retinal spots of 5 and $3 \mathrm{~mm}$. diameter respectively. Calibrations to 0.5 and $0.13 \mathrm{mV}$ inserted. Duration of stimulus on second beam of cathode ray is given below each record.

the stimulus. This is best seen by comparing the electroretinograms at equal levels of intensity: at 246 lux, for instance, the shape of the $b$-wave was much more influenced by a restriction of area than at the maximum intensity, while at 64 lux there was also a considerable difference in the size of potential. Over the whole range of areas stimulated at higher levels of intensity, the $b$-wave exhibited wavy contours. The increased amplification makes the results at low intensities less certain from this point of view. 
$2 \mathrm{~mm}$. retinal spot

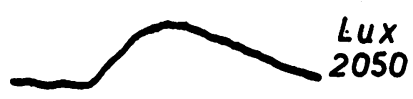

Mn

$50 \mathrm{cy} / \mathrm{sec}$.

FIG. 2.-Electroretinograms recorded at maximum intensity with retinal areas of 2 and $1 \mathrm{~mm}$. diameter respectively.
$1 \mathrm{~mm}$. retinal spot

$[0.13 \mathrm{mv}$

When smaller areas (1-2

when the intensity of the stimulus diminishes. change is very marked (Fig. 1).

The size and rate of rise of the $b$-wave was regularly measured in all experiments. In Fig. 3 the size of the $b$-wave from one experiment is plotted against the logarithm of intensity for filled pupil and for the different areas. Except for the smaller areas the S-shaped curve corresponds well with that previously obtained by Chaffee, Bovie, and Hampson (1923, on frogs), Hartline (1925, on man), and Granit (1933, on cats).

In Fig. 4(opposite) the height of the $b$-wave (per cent. of the maximum) is plotted against area. The broken line refers to the average potential at maximum intensity obtained from six long-lasting experiments and the full line to the results of a singleindependentexperiment in which the area only was changed, the maximum intensity being used throughout. The six experiments involved tests with several variables. The agreement between the two curves is good. There is a steep rise up to around $7 \mathrm{~mm}$. ${ }^{2}$ (3 mm. diameter); for greater

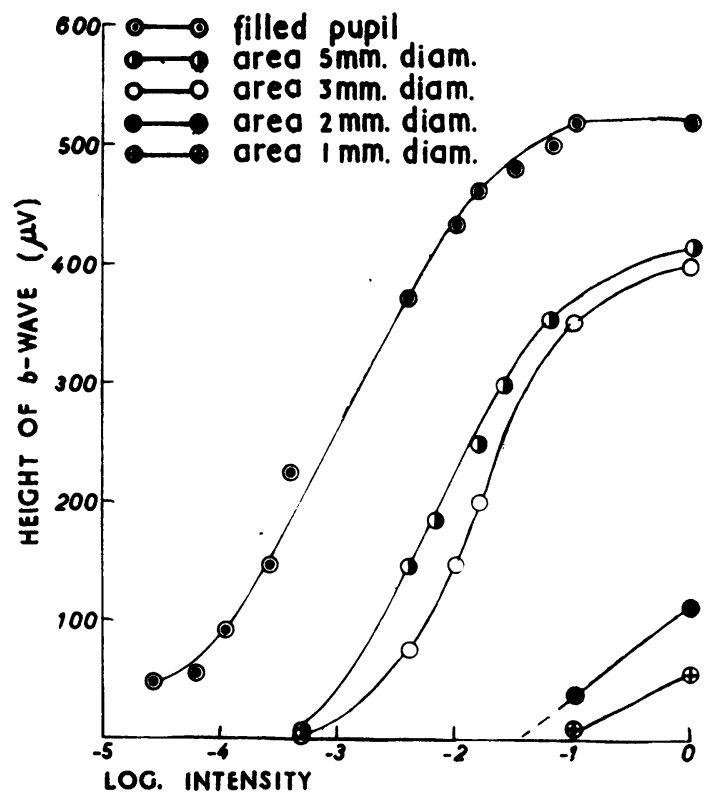

FIG. 3.-Height of $b$-wave from one experiment plotted against log. intensity for filled nupil, and areas of $5,3,2$ and $1 \mathrm{~mm}$. diameter.

areas the rise is slower. The difference in the range from $7 \mathrm{~mm} .^{2}$ to completely filled pupil (here marked $\infty$ ) is only about 24 per cent. If we assume the same relation to be roughly valid for the human electroretinogram and take the mean value of the scotopic $b$-wave as $0.36 \mathrm{mV}$ (Karpe, 1946), then for an area of $7 \mathrm{~mm} .{ }^{2}$, that is at the knee in the curve, one should obtain $0.27 \mathrm{mV}$, a value well within the limits of normal variation. If the rate of rise is plotted against 


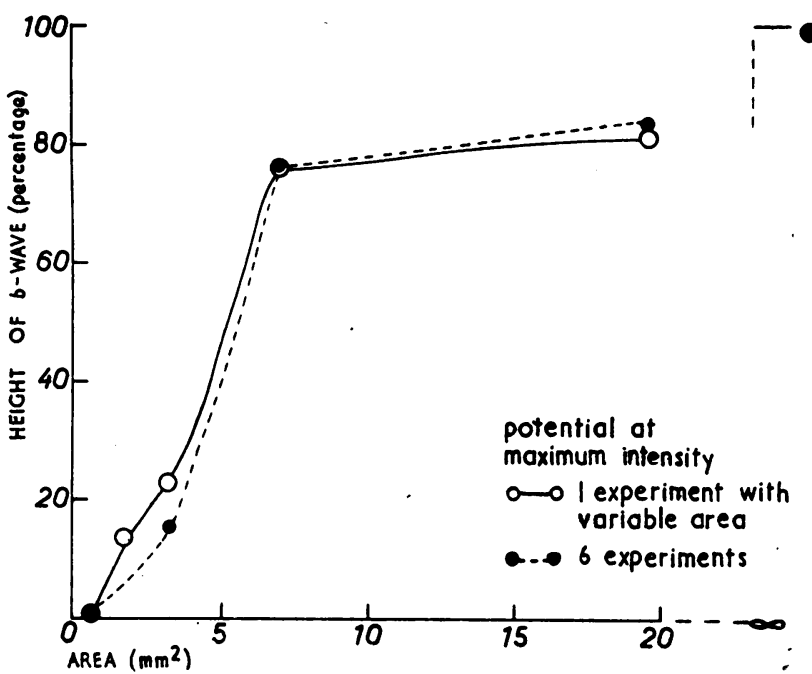

FIG. 4.-Height of $b$-wave as percentage of maximum plotted against area. area (Fig. 5, below), the curve is very different. From $7 \mathrm{~mm}^{2}$ up to completely filled pupil it is of the order of 63 per cent. We shall deal with this point below.

To find some expression for the variations in the shape of the electroretinogram, the rate of rise was calculated. This function is the relation between the height of the $b$-wave and the socalled peak time (time interval from the beginning of the $b$-wave to its peak). The results from the six experiments were averaged and plotted (full lines in Fig. 6, overleaf), the ordinates (on the left) representing the rate of rise in $\mathrm{mm}$./sec., and the abscissae the logarithm of intensity. The difference in the slope of the rate of rise of the curves is striking at high intensities, but, as Fig. 1 showed, the size of the $b$-wave did not vary a great deal with intensity at these levels. The $b$-waves merely became steeper when the light filled the whole pupil. At low intensities, however, the rate of rise was determined both by the size and the peak time of the potential. This is also emphasized by the shape of the curves relating amplitude of the $b$-wave (ordinates on the right) to log. I, plotted in the same figure(brokenlines),showing how they depart at high intensities.

The effect of area on flicker fusion was tested by the resonance method developed by Granit and Wirih (1953). Leads from the amplifier were taken both to the vibration analyser and to the

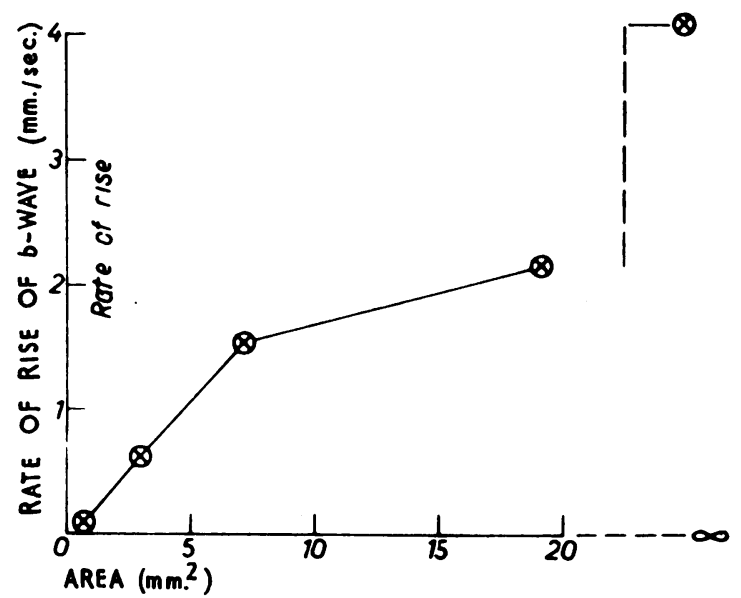

FIG. 5.-Relative rate of rise of $b$-wave plotted against area. cathode-ray oscillograph. In order to have a ready check on the precise coincidence of frequency in analyser and flickering beam, the output from the photocell (which signals the flashes on the cathode-tube) was connected as 
an alternative input to the vibration analyser. By watching its indicator needle with the photocell connected, the Velodyne potentiometer could be set for the required frequency and kept running at a constant rate. Then brief periods of illumination (3-4 sec. duration) were given as tests, and the

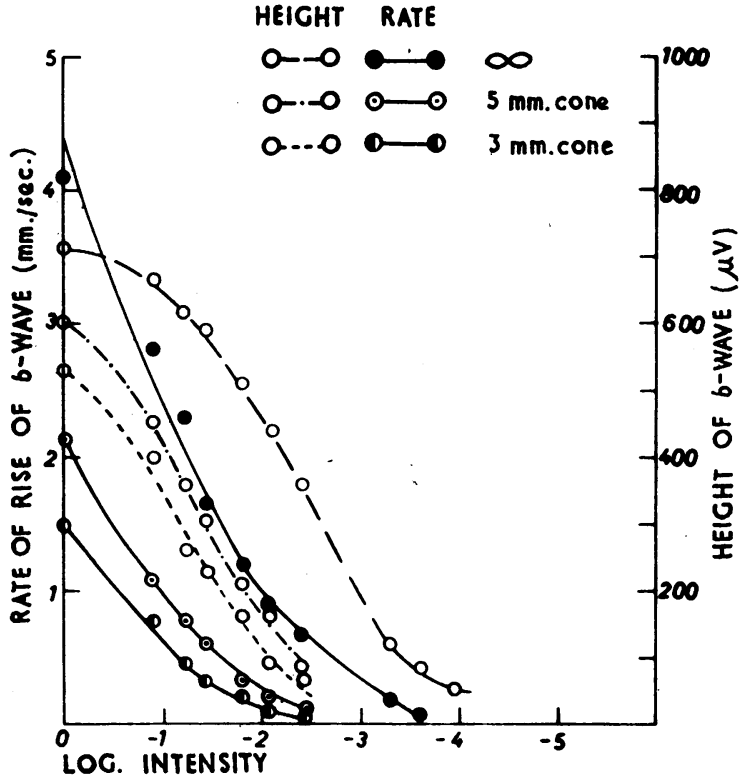

Fig. 6.-Relation between stimulus intensity and (1) rate of rise of $b$-wave (mm./sec.) (2) height of $b$-wave. Mean of six experiments. deflexion of the indicator needle of the resonance meter was observed. The frequency was varied accordingly, and fusion was taken as the point at which no deflexion (i.e. no resonance) could be recorded. When the $3 \mathrm{~mm}$.-cone was at the pupil so that the retina was diffusely illuminated, the fusion frequency was 73 flashes per sec. (intensity = 1,850 lux), but at the retinal surface the fusion fell to $14 \cdot 3$ flashes per second. This is in general agreement with the original observation by Granit and Harper (1930) on the effect of area in subjective flicker: over a large range of intensities an increase of stimulated area significantly augmented the fusion frequency.

\section{Discussion}

It is perfectly clear that small sharply restricted areas do not give small electroretinograms of the high intensity type such as are recorded when the whole retina is stimulated. The very striking effect of area upon flicker fusion is difficult to understand unless some integration takes place when the number of active neurones increases. It seems naturally related to the large changes in rate of rise due to area, as shown by Fig. 5. In the simple retina of Limulus the electroretinogram is affected in the same way by increasing the area as by increasing the intensity of the stimulus, but, as Graham (1932) showed, the size of the electroretinogram is simply proportional to area over a wide range of intensities, i.e., to the number of ommatidia stimulated; hence interaction need not be assumed in this case. In the cat's eye the size of the electroretinogram is roughly proportional to area when this is less than $7 \mathrm{~mm}^{2}$, but the rate of rise and fall of the $b$-wave enables one to distinguish electroretinograms obtained from small areas from those obtained from large areas.

Recently Kuffler (1953), using the microelectrode technique, found receptive fields in the cat's eye of the order of $3-4 \mathrm{~mm}$. diameter, a figure 
which is in agreement with the present results, although Kuffler was in doubt about the spread of light. This means that integration may take place within areas of the same order of magnitude as those found by electroretinography.

Considering again the records presented in Fig. 1, it is evident that, as far as the earlier phase of the retinal potential is concerned, both the definite $a$-wave and the initial faster component of the $b$-wave are present only in the case of filled pupil. This means that the electroretinogram is then of a more cone-like type since, apart from the $a$-wave, the faster component of the $b$-wave is related to photopic activity. Adrian (1946), using monochromatic light, demonstrated that the initial faster rise is connected with cone activity, a fact which has been since confirmed by a number of authors (Riggs, Berry, and Wayner, 1949; Armington, 1952; Schubert and Bornschein, 1952). When the area is decreased the electroretinogram at maximum intensity changes from a cone-like type to the usual rod type. This may indeed be due, in our case, to the fact that the cat's retina is dominated by rods, so that the area illuminated has to be very large in order to give the cones a chance of influencing the average response.

From the point of view of clinical electroretinography some points are of interest. As a rule, the electroretinogram recorded for practical purposes is the response of the whole retina, to which both photopic and scotopic components contribute depending on the intensity of the light. In the intact eye it is impossible to produce sharply focused areas, since its optical system is far from perfect. The fact that a field of definite size has to be stimulated in order to give a retinal potential of definite amplitude and shape obviously means that a certain population of retinal units must be activated. It is a common observation in clinical electroretinography that the $b$-potential may be of normal amplitude in spite of pathological conditions of the retina. As a rule records are taken with one or two intensities only; sometimes the peak time is measured, but generally the rate of rise is not taken into account. Yet it is clear from our results that, within a considerable range of intensities, the size of the $b$-wave varies but little, whilst its rate of rise or peak time is still greatly influenced by changes within that range. Peak time is therefore likely to give more information than measurement of the size of the $b$-wave alone. The advantage of measuring the $b$-wave over a larger range has been emphasized by Burian (1953), who in one patient with unilateral glaucoma found the $b$-wave much more susceptible to reduction of intensity in the glaucomatous eye than in the normal one.

On the basis of the present results it is tempting to calculate the size of the area that must be stimulated in order to produce a detectable electroretinogram in man. This should indicate approximately the limit of sensitivity of the method. In the cat the average maximum potential developed is $0.75 \mathrm{mV}$ when the light fills the pupil. With the $1-\mathrm{mm}$. cone the size of the $b$-wave, in the very best animals, is only 7 per cent. of the maximum, that is $0.052 \mathrm{mV}$. Assuming that the same relation holds for the human electro- 
retinogram and taking the mean maximum value to be $0.36 \mathrm{mV}$, one would expect $0.025 \mathrm{mV}$ for a receptor population functionally equivalent to an area of $0.78 \mathrm{~mm}^{2}$. A potential of this order cannot be measured even if the base-line is perfectly steady.

Another point of interest in this connection is concerned with perimetry by electroretinography, which Monnier and Boehm (1947) claim to be possible. They stimulated various points in the retinal field using an area of $2^{\circ}$ visual angle and a light intensity of $0 \cdot 1$ lumen. This means that, theoretically, the retinal area was less than $1 \mathrm{~mm}^{2}$. They found that the amplitude of the $b$-wave was higher, the peak time shorter, and the $a$-wave more evident, as the stimulus was moved towards the centre of the visual field (from $60^{\circ}$ to $20^{\circ}$ ). The size of the $b$-wave, however, was, on the whole, of the same order of magnitude as that obtained with non-localized stimuli. While their result probably signifies that the number of cones increases towards the centre, it nevertheless seems difficult to understand how the stimulus could have been restricted to the area under investigation, since the maximum potential the retina is likely to be able to develop with an area of $0.78 \mathrm{~mm} .{ }^{2}$ would be only about $0.02-0.03 \mathrm{mV}$, provided that maximum intensity were used. The results obtained by Boynton and Riggs (1951) and Asher (1951) with stimulation of the blind spot indirectly support this view. As already pointed out (Karpe, 1945; Wirth, 1950), electroretinographic perimetry would require threshold stimuli so as to avoid stray light effects at high intensities. It is, however, practically impossible to stimulate a restricted area with threshold intensity, since, as stated above, the potential is unlikely to be measurable even at maximum intensity. Admittedly our results were obtained with the cat's eye, but the human eye is not likely to be fundamentally different in this respect.

\section{Summary}

(1) A special device with Perspex cones, blackened on the outside and applied directly to the retina, was used to avoid stray light effects. Sharply focused spots of different diameters were thus produced in the opened cat's eye.

The sizes and shapes of the electroretinograms with standard leads were studied over a wide range of intensities for different areas.

Fusion frequency was measured by a resonance method recently described.

(2) In order to obtain an electroretinogram showing the haracteristic features of the high-intensity response, it was necessary to üsf cones with a diameter of 3-5 mm. With smaller areas the response assumed the characteristics of the low-intensity electroretinogram. It was impossibic to compensate for this effect of area by an increase of intensity.

In the best animals, a very small, slow response could be obtained with an area $1 \mathrm{~mm}$. in diameter, but normally the lower limit was between 1.5 and $2 \mathrm{~mm}$., and below this limit no electroretinogram could be recorded. 
(3) Curves illustrating size and rate of rise of the $b$-wave as a function of area and intensity are given.

(4) Only high-intensity responses, elicited by large areas, could produce high-fusion frequencies in "flicker".

(5) The significance of area is discussed with special reference to clinical electroretinography.

\section{REFERENCES}

Adrian, E. D. (1946). J. Physiol. (Lond.), 105, 24.

and MatThews, R. (1928). Ibid., 65, 273.

Armington, J. C. (1952). J. opt. Soc. Amer., 42, 393.

Asher, H. (1951). J. Physiol. (Lond.), 112, 40, p.

BOYNTON, R. M., and Riggs, L. A. (1951). J. exp. Psychol., 42, 217.

Burian, H. M. (1953). Arch. Ophthal. (Chicago), 49, 241.

Chaffee, E. L., Bovie, W. T., and Hampson, A. (1923). J. opt. Soc. Amer., 7, 1.

Creed, R. S., and Granit, R. (1933). J. Physiol. (Lond.), 78, 419.

ENROTH, C. (1952). Acta physiol. scand., 27, Suppl. 100.

Fry, G. A., and Bartley, S. H. (1935). Amer.J. Physiol., 111, 335.

Graham, C. H. (1932). J. cell. comp. Physiol., 2, 295.

Granit, R. (1933). J. Physiol. (Lond.), 77, 207. and Harper, P. (1930). Amer. J. Physiol., 95, 211, 229. Rubinstein, B., and Therman, P. O. (1935). J. Physiol. (Lond.), 85, 34 p. and WIRTH, A. (1953). Ibid., 122, 386.

Hartine, H. K. (1925). Amer. J. Physiol., 74, 600.

KARPE, G. (1945). Acta ophthal. (Kbh.), Suppl. 24,

KuffLer, S. W. (1953). J. Neurophysiol., 16, 37.

MONNIER, M., and BoEHM, F. (1947). Helv. physiol. pharmacol. acta, 5, 205.

Riggs, L. A., Berry, R. N., and WAyner, M. (1949). J. opt. Soc. Amer., 39, 427.

SCHUBERT, G., and Bornschein, H. (1952). Ophthalmologica (Basel), 123, 396.

WirTh, A. (1950). Atti Soc. Oftal. ital., 12, 199. 